\title{
Two-Stage Hybrid Repair in a Patient with Acute Type A Aortic Dissection Associated with Right Aortic Arch with Aberrant Left Subclavian Artery Originating from a Kommerell Diverticulum
}

\author{
Toshio Doi, MD, ${ }^{1}$ Kimimasa Sakata, MD, ${ }^{1}$ Takayuki Gyoten, MD, ${ }^{1}$ Saori Nagura, MD, ${ }^{2}$ \\ Akio Yamashita, MD, ${ }^{2}$ Kazuaki Fukahara, MD, ${ }^{2}$ Keiju Kotoh, MD, ${ }^{3}$ and Naoki Yoshimura, MD $^{2}$
}

Right aortic arch with aberrant left subclavian artery and Kommerell diverticulum are rare anomalies. A 42-year-old man was referred with sudden-onset chest pain. Enhanced computed tomography (CT) showed a right aortic arch with early thrombosed acute type A aortic dissection and an aberrant left subclavian artery arising from a Kommerell diverticulum. Medical therapy was instituted; however, follow-up CT revealed an ulcer-like projection. The patient was managed with a twostage hybrid procedure comprising total arch replacement and endovascular repair and experienced no postoperative complications. Two-stage hybrid repair is a safe and effective surgical option for rare complex aortic anomalies.

Keywords: two-stage hybrid repair, right aortic arch with aberrant left subclavian artery, Kommerell diverticulum

\section{Introduction}

Right aortic arch with aberrant left subclavian artery is a congenital anomaly found in approximately $0.1 \%$ of the general population. ${ }^{1)}$ Kommerell diverticula are aneurysms occurring at the origin of the aberrant subclavian artery. They are at risk of rupturing, a situation sometimes complicated by acute aortic dissection, and thus, surgical

${ }^{1}$ Department of Cardiovascular Surgery, JA Nagano Koseiren Shinonoi General Hospital, Nagano, Nagano, Japan

${ }^{2}$ Department of Thoracic and Cardiovascular Surgery, University of Toyama, Toyama, Toyama, Japan ${ }^{3}$ Department of Thoracic and Vascular Surgery, Toyama City Hospital, Toyama, Toyama, Japan

Received: February 14, 2016; Accepted: June 17, 2016 Corresponding author: Toshio Doi, MD. Department of Cardiovascular Surgery, JA Nagano Koseiren Shinonoi General Hospital, 666-1 Shinonoiai, Nagano, Nagano 388-8004, Japan

Tel: +81-26-292-2261, Fax: +81-26-2934-0025

E-mail: d-toshio@kg8.so-net.ne.jp management is critical for preventing fatal complications. Open-graft replacement with or without the reconstruction of the aberrant subclavian artery has been the standard procedure; however, hybrid surgical/endovascular repair has been recently reported. ${ }^{2)}$ We report on a patient with a right aortic arch with aberrant left subclavian artery originating from a Kommerell diverticulum complicated by acute type A aortic dissection in whom successful twostage hybrid total arch replacement and thoracic endovascular aortic repair (TEVAR) were performed.

\section{Case Report}

A 42-year-old man with a history of hypertension and hyperlipidemia was taken by ambulance to our emergency department with sudden-onset chest and back pain. His blood pressure was $134 / 87 \mathrm{mmHg}$, and his heart rate was $80 \mathrm{bpm}$ and regular. He was alert and moving all extremities. An electrocardiogram showed normal sinus rhythm with no ST-T segment change. Transthoracic echocardiography revealed normal left ventricular contraction and no valvular disease or pericardial effusion. Contrast-enhanced computed tomography (CT) showed early thrombosed acute type A aortic dissection between the right-sided ascending aorta and the abdominal aorta (Fig. 1A). The aortic dissection involved the wall of the Kommerell diverticulum. The aortic arch was routed from the tracheal and the esophageal right side to the posterior side of the trachea and the esophagus. Three-dimensional CT (3D-CT) demonstrated a right aortic arch with aberrant left subclavian artery arising from a $7-\mathrm{cm}$ Kommerell diverticulum (Fig. 1B). The right and left common carotid arteries arose from the anterior portion of the arch; the right subclavian artery arose more posteriorly from a dilated section of the arch, and the aberrant left subclavian from the large leftsided Kommerell diverticulum. No vascular compression of the esophagus or trachea was noted nor was the artery of Adamkiewicz visible. Thus, the diagnosis was a right 


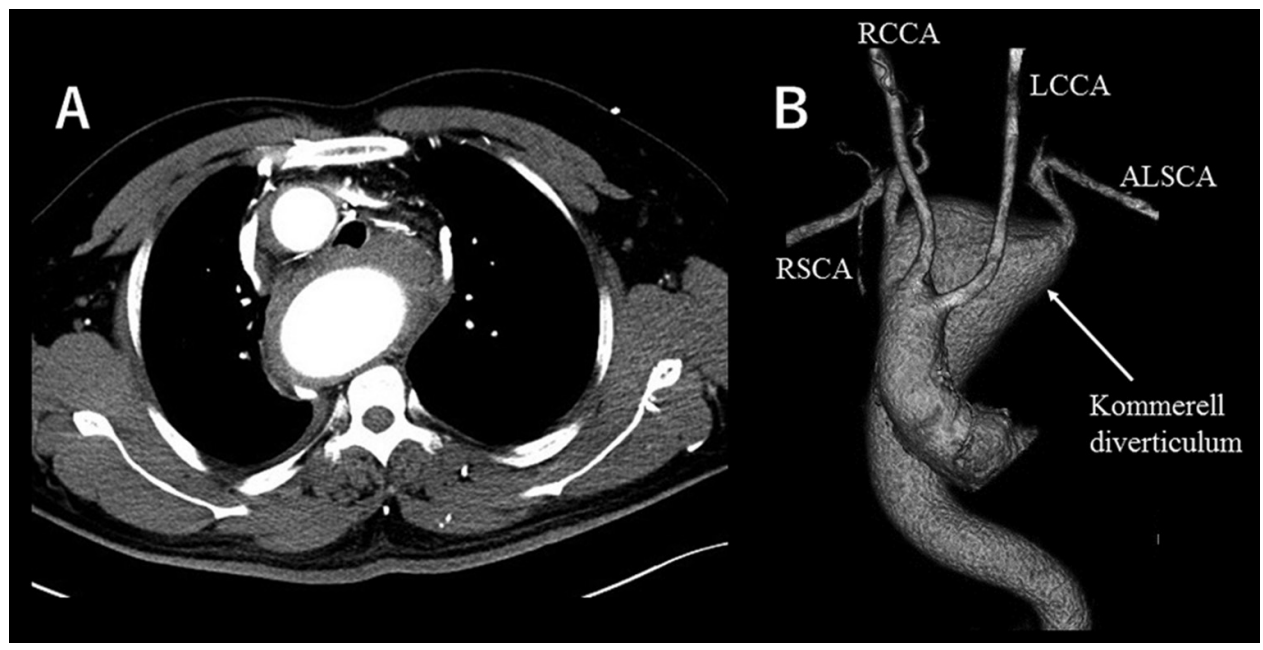

Fig. 1 (A) Preoperative computed tomography showing acute type A aortic dissection, which has thrombosed completely. (B) Preoperative three-dimensional computed tomography showing a rightsided aortic arch and aberrant left subclavian artery with a large Kommerell diverticulum (white arrow). LCCA: left common carotid artery; RCCA: right common carotid artery; RSCA: right subclavian artery; ALSCA: aberrant left subclavian artery

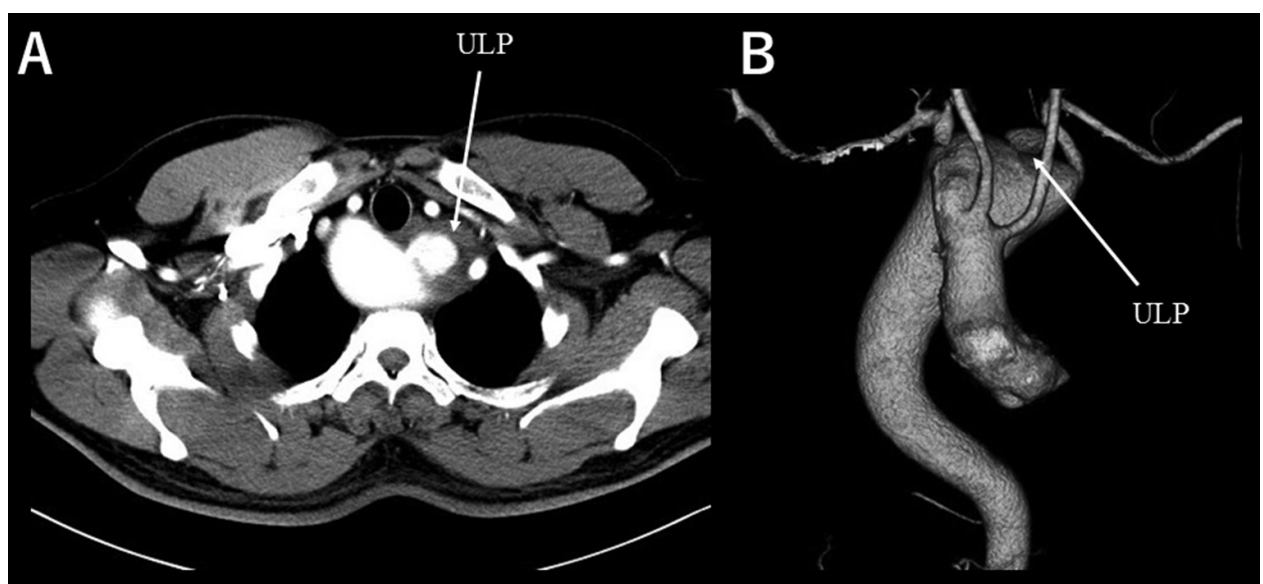

Fig. 2 (A) Preoperative follow-up enhanced computed tomography revealing ulcer-like projection at the aortic arch (white arrow). (B) Preoperative three-dimensional computed tomography revealing ulcer-like projection near the proximal site of the Kommerell diverticulum (white arrow). ULP: ulcer like projection

aortic arch with aberrant left subclavian artery arising from a Kommerell diverticulum associated with acute type A aortic dissection. Because the false lumen was completely thrombosed, aggressive medical therapy was initially instituted. A follow-up enhanced CT after 7 days revealed an ulcer-like projection (ULP) entering the thrombus in the aortic arch (Figs. 2A and 2B); thus, urgent surgery was planned. We chose a two-stage hybrid surgical repair approach, consisting of total arch replacement using the frozen elephant trunk (FET) tecnique, followed by a TEVAR as a less invasive surgical option. We planned the distal anastomosis site for total arch replacement to be on the right side of the trachea using FET because the aortic arch was posterior to the trachea.

The first stage was performed via median sternotomy. After systemic heparinization, cardiopulmonary bypass (CPB) was established with arterial cannulas in the left femoral artery; 8-mm graft conduits were anastomosed to the axillary arteries in an end-to-side fashion via bilateral infrasubclavian incisions and venous drainage from bicaval cannulation. Left ventricular venting was performed, and systemic cooling was performed to decrease the rectal temperature to $25^{\circ} \mathrm{C}$. The ascending aorta was crossclamped and resected just above the sinotubular junction. 


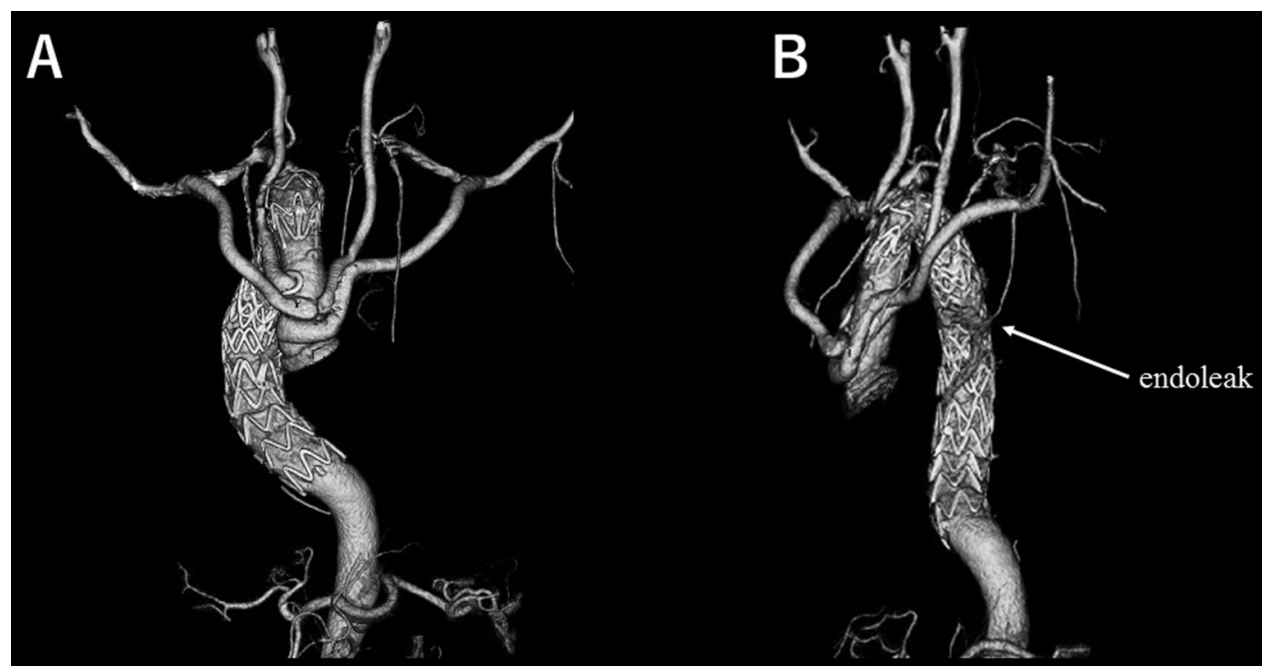

Fig. 3 (A) Postoperative three-dimensional computed tomography (anterior view) demonstrating patent reconstructed supra-aortic vessels. (B) Postoperative three-dimensional computed tomography (left anterior oblique view) demonstrating a minor type II endoleak from a small branch of the left subclavian artery (white arrow).

The crystalloid cardioplegic solution was used for cardiac arrest in a selective antegrade fashion.

We identified a dissected ascending aorta to aortic root with a thrombosed false lumen. After the removal of the thrombus from the false lumen, a bioadhesive (BioGlue; Cryolife International, Inc., Kennesaw, GA, USA) was used to obliterate the false lumen. The proximal ascending aortic stump was anastomosed to the proximal end of a 24-mm quadrifurcated graft (J graft; Japan Lifeline Co., Ltd., Tokyo, Japan) using a 4-0 polypropylene running suture with Teflon felt strip reinforcement of the outside of the aortic wall. Under circulatory arrest, the aortic clamp was removed. Both common carotid arteries were resected, and the right subclavian artery was ligated at its origin. The left subclavian artery was temporarily occluded at the origin by an occlusion balloon catheter inserted into the left brachial artery. Antegrade selective cerebral perfusion (total flow, $12 \mathrm{~mL} / \mathrm{kg} / \mathrm{min}$ ) was established through the 8 -mm graft conduits anastomosed to the axillary arteries, and the perfusion balloon catheters were inserted directly into the common carotid arteries. The aortic arch was completely transected immediately distal to the origin of the right subclavian artery. The distal aortic stump was reconstructed by the FET technique using a $27 \times 90$-mm stent graft (J Graft Open Stent Graft; Japan Lifeline, Co., Ltd., Tokyo, Japan) on the right side of the trachea and was anastomosed to the distal end of the quadrifurcated graft. After air removal, CPB and systemic warming were restarted. The common carotid arteries were anastomosed to the side branches of the quadrifurcated graft in an endto-end fashion, and the subclavian arteries were reconstructed by anastomosing 8 -mm graft conduits to the side branches of the quadrifurcated graft in an end-to-end fashion. The patient was uneventfully weaned from CPB. The time of CPB, cardiac arrest, and circulatory arrest was 200, 99, and $65 \mathrm{~min}$, respectively. The postoperative course was uneventful except for transient hoarseness.

The second stage, including TEVAR and left subclavian artery embolization, was performed 14 days after the first operation. The left common femoral artery was exposed as an access route. A $32 \times 200$-mm stent graft (Relay plus; Bolton Medical Inc., Barcelona, Spain) was deployed immediately distal to the origin of the last side branch that was anastomosed to the right subclavian artery. Next, a second $34 \times 200-\mathrm{mm}$ stent graft (Relay plus; Bolton Medical Inc., Barcelona, Spain) was deployed to exclude the Kommerell diverticulum, with the stent grafts overlapping. Finally, the origin of the left subclavian artery was embolized by a 12-mm plug (Amplatzer Vascular Plug II; St. Jude Medical Inc., St. Paul, MN, USA) and coils (Tornado Embolization Coil; Cook, Bloomington, IN, USA) by a left brachial-artery approach. The patient experienced no postoperative paraplegia. Postoperative 3D-CT demonstrated that the bypass grafts were patent and that the Kommerell diverticulum was excluded by the stent grafts with a minor type II endoleak from a small branch of the left subclavian artery (Figs. 3A and 3B).

\section{Discussion}

Kommerell diverticulum is believed to be an embryologic remnant of the dorsal fourth aortic arch. ${ }^{3)}$ Although patients with Kommerell diverticulum and an aberrant subclavian artery are usually asymptomatic, chest pain, cough, 
respiratory discomfort, dysphagia, and other symptoms may occur. ${ }^{4)}$ However, surgical intervention can relieve these symptoms. Several studies have examined the morbidity/mortality of asymptomatic aberrant subclavian arteries with Kommerell diverticulum. Austin et al. reported that $19 \%$ of patients with Kommerell diverticula developed diverticular rupture and died, ${ }^{5)}$ and Cina et al. reported that the incidence of diverticular rupture in patients with Kommerell diverticula was $6 \%{ }^{4}{ }^{4}$ Kouchoukos et al. found diverticular dissection in 2 of 10 patients, ${ }^{6}$ and Indrees et al. reported an incidence of diverticular dissection of $50 \% .^{7)}$ Hence, because asymptomatic Kommerell diverticula are at substantial risk of rupture and dissection, surgery is indicated regardless of the presence or absence of symptoms.

However, determining the necessity of surgery solely on the basis of Kommerell diverticulum size is challenging. Cina et al. reported that the mean diameter of ruptured Kommerell diverticula was $5.8 \mathrm{~cm} \pm 2.0 \mathrm{~cm}$ and that because surgery becomes more complicated with increasing diverticulum diameter, surgery should be applied only if the diameter exceeds $3 \mathrm{~cm}^{4}{ }^{4}$ Tanaka et al. reported that the mean Kommerell diverticulum diameter was $4.6 \mathrm{~cm} \pm 1.3 \mathrm{~cm}$ when the rupture occurred and $4.6 \mathrm{~cm} \pm 2.0 \mathrm{~cm}$ when dissection was performed. ${ }^{8}{ }^{8}$ In the present case, we judged that surgery was indicated, given the acute type A aortic dissection with ULP and the large Kommerell diverticulum.

There is no definitive surgical method available for treating a Kommerell diverticulum with a right aortic arch. Resection of aneurysms of the aorta, including aortic diverticula, graft replacement, and aberrant subclavian artery reconstruction, are usually performed together. However, these single-stage surgical methods require a large posterolateral thoracotomy and deep hypothermic circulatory arrest and are markedly invasive, ${ }^{4,5)}$ with a risk of respiratory complications and spinal cord ischemia. Austin et al. reported that the operative mortality associated with these surgical methods was $16.6 \%$, and Cina et al. reported an operative mortality of $12 \%$ and an incidence of paraplegia of $4 \% .45$ According to Kouchoukos et al., the operative mortality for elective surgery was $0 \%$, although the number of patients undergoing elective surgery was small in their study. ${ }^{6}$ Tanaka et al. reported that the early operative mortality associated with surgery for Kommerell diverticulum was $11 \%$ and that the causes of death included acute pulmonary embolism, multiple organ failure, sepsis, and hemorrhage. ${ }^{8)}$ Although postoperative outcomes are improving, one-stage, widerange open-graft replacement remains unsafe.

TEVAR has been reported to be a useful, less-invasive treatment for right aortic arch-associated Kommerell diverticulum. ${ }^{2)}$ Because anatomical details differ among patients, endovascular treatment for this disease needs to be individually developed. Usually, an endovascular treatment is performed in combination with surgery, such as cervical branch bypass. Idrees et al. reported good outcomes after three types of hybrid surgery. ${ }^{7)}$ However, because TEVAR is unlikely to improve acute symptoms in patients who have pressure symptoms from the diverticulum, conventional aneurysmectomy and graft replacement are often necessary. Determining whether or not TEVAR should be performed requires careful assessment of preoperative symptoms and anatomical investigation. 3D-CT is useful for visualizing the course of the aorta and cervical branches and positional relationships with their surrounding organs and for establishing a treatment plan. Another issue with TEVAR is the risk of endoleaks. Although no particular treatment is deemed necessary for type II endoleaks, as most spontaneously disappear or do not worsen aneurysms, close follow-up is essential..$^{9}$ The patient we treated developed a small type II endoleak from the branch of the left subclavian artery and was requested to visit our hospital regularly for a close follow-up.

Our patient had an acute type A aortic dissection and an aberrant left subclavian artery arising from a $7-\mathrm{cm}$ Kommerell diverticulum, which required extensive graft replacement from the ascending to the descending thoracic aorta. Given the patient's extensive abnormalities, we discussed whether one-stage or two-stage surgery should be performed. Although one-stage surgery is preferable, it is invasive and involves a high risk of complications. We finally selected a two-stage surgery consisting of a total arch replacement using the FET technique and TEVAR. In this case, usual total arch replacement was very difficult because the aortic arch routed posterior to the trachea; however, the use of the FET technique involving a J-Graft open stent made the distal anastomosis procedure easier. We chose a short stent graft because using a long frozen elephant trunk represented a risk factor for the patient developing paraplegia. We confirmed that the distal end of the $90-\mathrm{mm}$ open stent was at the Th6 level and that the Kommerell diverticulum was not completely excluded by the open stent during the second-stage procedure. The $\mathrm{J}$ graft open stent allowed easier distal anastomosis of the total arch replacement and the development of an adequate proximal landing zone for TEVAR. However, although two-stage surgery is less invasive, residual vascular lesions might rupture between the two stages, and the second procedure must be performed as soon as possible after the first surgery. Safi et al. reported that in patients undergoing two-stage surgery for extensive aortic aneurysms, the incidence of residual aortic aneurysm rupture between the two stages was $16 \%{ }^{10)}$ In our patient, we performed a TEVAR as the second procedure 14 days after the first surgery. Although we could have performed the surgery earlier, we decided that it would be safer to delay the second procedure so as to allow the patient's physical condition and hemodynamic status to stabilize to prevent 
postoperative paraplegia because we could not have identified the Adamkiewicz artery preoperatively.

\section{Conclusion}

Right aortic arch with aberrant left subclavian artery originating from a Kommerell diverticulum complicated by type A aortic dissection is very rare, and the treatment strategy for this condition needs to be based on the anatomy of each individual. 3D-CT is very useful in these cases. The two-stage hybrid repair approach comprising total arch replacement with FET technique and TEVAR might be a useful surgical strategy for complex aortic diseases that can help avoid postoperative complications, such as paraplegia.

\section{Disclosure Statement}

The authors have no conflicts of interest to disclose in relation to this study.

\section{Author Contributions}

Study conception: TD

Data collection: TD, KS

Analysis: TD, KS

Writing: TD

Clinical review and revision: all authors

Final approval of the article: all authors

Accountability for all aspects of the work: all authors

\section{References}

1) Hastreiter AR, D’Cruz IA, Cantez T, et al. Right-sided aorta. I. Occurrence of right aortic arch in various types of congenital heart disease. II. Right aortic arch, right descending aorta, and associated anomalies. Br Heart J 1966; 28: 722-39.

2) Kawajiri H, Shimizu H, Yoshitake A, et al. Hybrid repair of a Kommerell diverticulum associated with a right aortic arch and a left descending aorta. J Vasc Surg 2012; 56: 1727-30.

3) Backer CL, Russell HM, Wurlitzer KC, et al. Primary resection of Kommerell diverticulum and left subclavian artery transfer. Ann Thorac Surg 2012; 94: 1612-7.

4) Cinà CS, Althani H, Pasenau J, et al. Kommerell's diverticulum and right-sided aortic arch: a cohort study and review of the literature. J Vasc Surg 2004; 39: 131-9.

5) Austin EH, Wolfe WG. Aneurysm of aberrant subclavian artery with a review of the literature. J Vasc Surg 1985; 2: 571-7.

6) Kouchoukos NT, Masetti P. Aberrant subclavian artery and Kommerell aneurysm: surgical treatment with a standard approach. J Thorac Cardiovasc Surg 2007; 133: 888-92.

7) Idrees J, Keshavamurthy S, Subramanian S, et al. Hybrid repair of Kommerell diverticulum. J Thorac Cardiovasc Surg 2014; 147: 973-6.

8) Tanaka A, Milner R, Ota T. Kommerell's diverticulum in the current era: a comprehensive review. Gen Thorac Cardiovasc Surg 2015; 63: 245-59.

9) Preventza O, Wheatley GH, Ramaiah VG, et al. Management of endoleaks associated with endovascular treatment of descending thoracic aortic diseases. J Vasc Surg 2008; 48: 69-73.

10) Safi HJ, Miller CC, Estrera AL, et al. Optimization of aortic arch replacement: two-stage approach. Ann Thorac Surg 2007; 83: S815-8; discussion S824-31. 